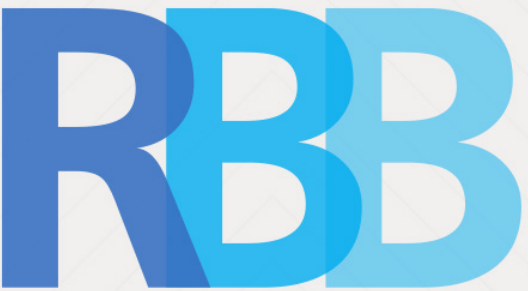

Revista Brasileira de Bioética

\section{Adriana Bottoni}

Programa de Pós-graduação em

Bioética, Centro Universitário São

Camilo, São Paulo, SP, Brasil

adriana.bottoni@icloud.com

Vera Lucia Zaher-Rutherford

Programa de Pós-graduação em

Bioética, Centro Universitário São

Camilo e Hospital das Clínicas,

Faculdade de Medicina da USP, São

Paulo, SP, Brasil

zaher@usp.br

\section{Reflexão Bioética sobre uso de nutrição e hidratação artificial em pacientes terminais}

\author{
Bioethical reflection on the use of nutrition and artificial \\ hydration in terminal patients
}

Resumo: As questões referentes à terminalidade da vida levantam muitas discussões no contexto da bioética. Os avanços tecnológicos na medicina têm permitido salvar a vida de doentes críticos, entretanto, naqueles sem possibilidade de recuperação, a utilização de medidas curativas, além de onerosas e sem benefícios, acabam causando dor e sofrimento. A nutrição e a hidratação artificiais são consideradas tratamento médico e o profissional da saúde deve tomar decisões de acordo com sua utilidade. Refletir sobre questões de alimentação relacionadas à finitude sempre provoca polêmicas e opiniões divergentes entre familiares e até mesmo entre os profissionais de saúde. Esse trabalho tem por objetivo, à luz da bioética, propor uma reflexão sobre a importância do cuidar quando a cura é impossível e reconhecer a terminalidade como processo humano, natural e necessário. Nutrir o paciente no fim de vida tem um significado mais simbólico do que fisiológico e no caso da demência avançada pode causar desconforto e piora clínica. A cultura e a religião do paciente também devem ser priorizadas nesta fase. O ideal é que a vontade do paciente terminal seja respeitada por meio das diretivas antecipadas de vontade, o que pode diminuir a sobrecarga emocional de familiares ou responsável legal no que tange a tomada de decisão. No fim de vida de pacientes com demência avançada, a prioridade deve ser proporcionar uma morte digna, respeitando os princípios de beneficência, não maleficências e justiça.

Palavras-chave: Terminalidade de vida, bioética, terapia nutricional, ortotanásia, autonomia, cuidados paliativos.

Abstract: Issues regarding the termination of life raise many discussions in the context of bioethics. Technological advances in medicine have saved the lives of critically ill patients; however, in those with no possibility of recovery, the use of curative measures, besides costly and without benefits, end up causing pain and suffering. Artificial nutrition and hydration are considered medical treatment and the health professional must make decisions according to their utility. Reflecting on feeding issues related to finitude always provokes controversies and divergent opinions between family members and even among health professionals. This work aims, in the light of bioethics, to propose a reflection on the importance of caring when healing is impossible and to recognize terminality as a human, natural and necessary process. Nurturing the patient at the end of life has a more symbolic than physiological meaning and in the case of advanced dementia can cause discomfort and clinical worsening. The culture and religion of the patient should also be prioritized at this stage. Ideally, the will of the terminal patient should be respected through anticipated directives of will, which may reduce the emotional overload of family members or legal guardians when it comes to decision making. At the end of life of patients with advanced dementia, the priority must be to provide a dignified death, respecting the principles of beneficence, nonmaleficence and justice.

Keywords: End of life, bioethics, nutrition terapy, orthotanasia, autonomy, palliative care. 


\section{Introdução}

Para o filósofo Friedrich Wilhelm Nietzsche, o que dá significado à morte é o amor à vida, sobretudo quando o homem toma consciência da sua finitude e passa a assumir, frente ao sofrimento, a responsabilidade em relação à própria existência (Nasser, 2008). Para Schopenhauer, a percepção do homem de que ele é finito é que o faz temer a morte. Tal evento encontrará o homem por caminhos diferentes, mas a religião ou a filosofia individual é que determinará como ele enfrentará a morte. Schopenhauer assume que algumas religiões não preparam o indivíduo deixando-o inseguro perante a morte dele mesmo ou de familiares e o sofrimento causado por ela é aceitável e esperado pela sociedade (Salviano, 2012).

A sociedade ocidental, frente ao desenvolvimento de novas técnicas no campo da medicina, passou a encarar a morte não mais como um evento metafísico, mas sim como um fracasso. Nesse contexto, faz-se necessário buscar a reinterpretação da morte. Com o avanço tecnológico, o prolongamento artificial da vida passou a ser uma realidade, produzindo sofrimento e desmotivação, não apenas aos familiares e amigos do paciente, como também nos profissionais de saúde envolvidos, uma vez que a formação desses profissionais se sustenta na busca do curar em detrimento do cuidar (Kovács, 2005). Diante desse cenário, urge a discussão sobre temas relacionados à morte e ao morrer: o testamento em vida, morrer com dignidade e a não utilização de meios que prolonguem a vida, como por exemplo a utilização da nutrição e hidratação artificiais (NHA).

Na medicina, o "fim da vida" é o período que precede a morte natural de um indivíduo, fruto de um processo que não pode ser evitado (Hui et al. 2014). A terminalidade se apresenta como uma realidade quando se esgotam as chances de cura do paciente e a possibilidade de morte próxima parece inevitável e previsível (Marengo et al. 2009). Aqui deve-se fazer distinção entre os conceitos: o termo "final da vida" é usado para definir um determinado período da vida do paciente, doente terminal é a descrição da condição do paciente e o cuidado terminal são os cuidados prestados a esses pacientes doentes (Hui et al. 2014). Segundo Knobel e Silva: "Paciente terminal é aquele que possui uma condição de saúde irreversível, independentemente de estar ou não sob tratamento, e que, com base nesse quadro, apresenta uma alta probabilidade de morrer num período relativamente curto de tempo" (2004). 
A NHA não deve necessariamente continuar nos pacientes na fase terminal. No entanto, ainda existem controvérsias e uma crença discordante na prática quando a nutrição artificial e a hidratação são retiradas. Isso se deve ao fato de que há mais valor emocional ligado à provisão de nutrição e hidratação do que, por exemplo, no uso contínuo de antibióticos ou outros tratamentos. É importante levar em consideração o princípio da autonomia do paciente. A decisão de fornecer NHA deve basear-se em evidências, melhores práticas, experiência clínica e julgamento; comunicação eficaz com o paciente, família e/ou tomador de decisão substituto autorizado; e respeito à autonomia e a dignidade do paciente. O paciente se torna vulnerável quando perde a consciência e consequentemente perde sua autonomia e a partir desse momento, a família ou representante legal torna-se responsável pela decisão de implementar ou não a nutrição e hidratação artificiais. Diante de todos esses aspectos, o presente trabalho tem por objetivo realizar uma revisão sobre as questões bioéticas e éticas que envolvem a nutrição e hidratação artificial e a terminalidade, abordando o papel cultural, as crenças e religiões e o papel do médico como provedor de um término de vida com qualidade e dignidade para esses indivíduos, respeitando o princípio básico da não maleficência.

\section{Material e métodos}

Este trabalho trata-se de uma revisão integrativa de literatura. Esse tipo de revisão constitui uma abordagem metodológica referente às revisões e inclui a análise de trabalhos relevantes que dão suporte para a tomada de decisão e a melhoria da prática clínica, possibilitando a síntese de vários estudos publicados e conclusões gerais a respeito de uma área de estudo em particular. Combina também dados da literatura teórica e empírica, além de incorporar um vasto leque de propósitos: definição de conceitos, revisão de teorias e evidências, e análise de problemas metodológicos de um tópico particular. A revisão produz conhecimento atualizado sobre determinado problema e determina se esse conhecimento pode ser aplicado na prática (Souza et al. 2010).

A busca dos periódicos se deu nas bases virtuais indexadas à Biblioteca Virtual em Saúde e teve como critério de inclusão os artigos publicados em periódicos nacionais e internacionais na língua inglesa, portuguesa e em espanhol utilizando-se descritores como: Autonomia pessoal, Bioética, Cuidados paliativos na terminalidade da vida, Demência, Direito a morrer, Terapia nutricional. As bases de dados utilizadas 
foram o PUBMED e SCIELO. Quanto aos critérios de exclusão, levaram-se em consideração: artigos em duplicidade; aqueles que, apesar de apresentarem os descritores selecionados, não abordavam diretamente a temática proposta; artigos em língua diferente de inglês, espanhol e português; artigos que não tinham abstract disponível e nem Doi. artigos que falavam desta temática em crianças neonatos e adolescentes.

Inicialmente utilizamos combinação de dois descritores, porém observamos que ou o numero de artigos era muito grande e com muito poucos artigos sendo selecionados, ou muito pequeno. Com o cruzamento de terapia nutricional, Cuidados paliativos, terminalidade da vida, foram encontrados 105 artigos e destes, 29 foram selecionados. Ao associar o descritor Bioética aos anteriores, somente 3 artigos foram selecionados e estavam incluídos na pesquisa anterior. Cruzando Bioética, Cuidados paliativos, terminalidade da vida e terapia nutricional, foram encontrados 20 artigos e desses, selecionados 8. Ao cruzar Bioética, Cuidados paliativos, terminalidade da vida 26 estudos foram encontrados e 8 selecionados. Sobre demência, Cuidados paliativos na terminalidade da vida e terapia nutricional como resultado foram obtidos 101 artigos e selecionados 35 . O descritor em inglês artificial nutrition associado à Cuidados paliativos, terminalidade da vida resultou em 238 artigos, dos quais 40 foram selecionados. $\mathrm{O}$ cruzamento dos descritores Cuidados paliativos na terminalidade da vida, terapia nutricional e autonomia pessoal, direito a morrer resultaram em 45 artigos e desses foram selecionados 9 . Já com o cruzamento de terapia nutricional e autonomia pessoal foram encontrados 65 artigos e selecionados 10 .

Os artigos selecionados foram revisados e os que estavam em duplicata descartados. Os guidelines e posições das diversas sociedades internacionais sobre a temática do presente trabalho foram incluídos. Estes artigos nortearam os principais temas discutidos ao longo desse trabalho, temas estes relevantes para uma análise bioética.

\section{Desenvolvimento}

A Bioética é um ramo da Ética que enfoca questões referentes à vida humana e, consequentemente, à morte, propondo questões relativas às mais variadas situações, inclusive referente ao processo de morrer com dignidade (Ricoeur, 2007; Zoboli e Schveitzer 2013). De acordo com Torres (Torres, 2003), a bioética propõe como princípios básicos a Justiça, a Autonomia, a Beneficência e a Não-Maleficência, e estes princípios se tornaram a base da ética profissional na área da saúde. A autonomia 
tem como ideal que a escolha seja feita pelo próprio paciente. A beneficência refere-se ao alívio do sofrimento do paciente a não-maleficência prega uma avaliação muito precisa dos benefícios e consequências de todas as terapias que poderão ser utilizadas nestes pacientes e a justiça tem por objetivo trazer conforto à vida restante desse paciente (Chernoff, 2006; Geppert et al. 2010; Palecek et al. 2010; Snyder et al. 2013).

É fundamental nesse contexto o reconhecimento da terminalidade como processo humano, natural e necessário e assim, retomar o pensamento de Hipócrates, que destaca como um dos papéis da medicina "recusar-se a tratar daqueles que foram vencidos pela doença, entendendo que, diante de tais casos, a medicina torna-se impotente" (Lima, 2006)

As questões referentes à terminalidade da vida levantam muitas discussões no contexto da bioética (Steinhauser et al. 2000; Moritz, 2005; Bitencourt et al. 2007). No processo da terminalidade deve-se levar em conta não a quantidade de vida que resta à pessoa, mas sim a qualidade de vida (Giacomin et al. 2013). Os avanços tecnológicos na medicina têm permitido salvar a vida de doentes críticos, entretanto, naqueles sem possibilidade de recuperação, a utilização de medidas curativas, além de onerosas e sem benefícios, acabam causando dor e sofrimento (Lago et al. 2007). No Brasil muito se discute sobre a legalização e a aplicação da ortotaná $\operatorname{sia~-~o~não~}$ prolongamento artificial do processo natural da morte, praticado apenas pelo médico, com o consentimento do paciente ou do familiar, como um meio de se alcançar a morte digna (Neto, 2010). A questão central ética e moral - nas decisões de limitar ou retirar as medidas de suporte a vida do paciente no processo da morte, é saber se a te rapêutica oferece esperança de recuperação.

A morte, desde o princípio da história, se dava quando as funções básicas do organismo cessavam. Com a evolução tecnológica e a manutenções das funções vitais por meio de equipamentos a morte foi postergada. A morte passou a ser entendida como um processo de morrer e não apenas como um momento isolado, evento que ainda é pouco aceito pela sociedade.

O entendimento sobre a morte pode ser diferente de acordo com a cultura, espiritualidade e religiosidade de cada indivíduo. Em um contexto mais amplo, a cultura não é apenas determinada por etnia ou raça; é determinado ou influenciado pela espiritualidade / religião, nível socioeconômico, nível de instrução, nível de aculturação, 
sexo, idade, orientação sexual, país de origem e status de imigração. A cultura é um conceito importante a ser considerado quando nos preocupamos com os doentes terminais, uma vez que ela influenciará a maneira como eles entendem sua doença e morte, bem como a forma como tomam decisões sobre o fim da vida. Porém, independentemente da formação cultural ou preferências, existem necessidades que são universais para todos os pacientes. Todos eles, bem como seus familiares desejam abordar o fim da vida com dignidade, respeito próprio e uma oportunidade para construir e garantir seu legado. $O$ que difere pode ser como cada indivíduo realiza essas tarefas (Smith et al. 2008; Mitchell e Mitchell 2009).

Smith e colegas (Smith et al. 2008) realizaram um estudo para avaliar o impacto do reconhecimento, religiosidade e preferências de tratamento da doença terminal nos cuidados paliativos. De acordo com seus achados, muitos dos pacientes que identificaram a espiritualidade e a religiosidade como importantes para sua experiência e resposta à doença, desejavam a oportunidade de discutir seus desafios espirituais com sua equipe de saúde. Um dado interessante é que alguns pacientes viam seus cuidadores como ferramentas utilizadas por Deus para tratar o sofrimento ou oferecer cura, porém a decisão final sobre o resultado de uma doença dependeria de Deus e, portanto, se a medicina falhasse, os milagres ainda seriam possíveis. A doença é frequentemente vista como "vontade de Deus" e a aceitação da "vontade de Deus" é frequentemente usada como uma estratégia de enfrentamento - aqueles que viram sua doença sob essa luz geralmente acreditavam que Deus estava no controle total do resultado da situação. A espiritualidade é frequentemente usada para ajudar a apoiar a aceitação de um diagnóstico. A religião também é capaz de lançar uma outra luz sobre a morte: enquanto os ateus acreditam que não exista nenhuma vida após a morte, as pessoas que carregam alguma crença acreditam em uma nova vida ou uma salvação após a morte, o que traria uma sensação de reconforto diante do inevitável.

Uma pesquisa realizada por Van Wijmen et al. mostrou uma probabilidade significativamente menor na recusa de ressuscitação de pacientes com demência avançada (47\%) e câncer (53\%) no grupo orientado para o cristianismo quando comparados com população em geral ( $86 \%)$. Pessoas sem afiliação religiosa eram mais propensas a recusar tratamento para uma doença terminal (2015).

O sistema legal judaico diferencia as ações ativas e passivas e, portanto, diferencia a retenção e a retirada de terapias que sustentam a vida. Para os judeus conservadores e ortodoxos, a prioridade é a preservação da vida e os tratamentos quando 
iniciados não podem ser interrompidos e sob este aspecto, nutrição artificial e a hidratação não podem ser recusadas. Os líquidos e alimentos são considerados como necessidades básicas e não como tratamento e retirá-los de um paciente vegetativo ou moribundo é considerado como uma forma de eutanásia. Porém, se a nutrição artificial e a hidratação causarem sofrimento ou complicações, é permitido retirá-los, caso seja vontade do paciente (Druml et al. 2016).

Em relação às religiões orientais (incluindo o hinduísmo e o budismo) o uso de medicamentos opiáceos no final da vida é negado a fim de se manter o nível de consciência no momento da morte. Além disso, o jejum é considerado uma fonte de purificação espiritual pelos hindus e, portanto, estes se opõem à alimentação por sonda na fase terminal da doença (Firth, 2005).

A lei islâmica permite a retirada de tratamento fútil, que retarda a morte, incluindo o suporte de vida, de pacientes terminais, permitindo que a morte siga seu curso natural. No entanto, a retirada deve ser uma decisão coletiva baseada no consentimento informado e deve ocorrer apenas quando os médicos estiverem certos de que a morte é inevitável e que o tratamento não melhorará a saúde ou a qualidade de vida do paciente (Alsolamy, 2014).

Um estudo realizado com idosos budistas da Tailândia revelou que a maioria queria saber a verdade sobre a sua doença e estar livre de sintomas desconfortáveis. Quando a chance de sobreviver era pequena, aproximadamente $75 \%$ dos entrevistados não queria tratamentos prolongados. Curiosamente, $56 \%$ por cento dos idosos revelou não querer morrer em casa (Srinonprasert et al. 2014).

Na maioria das religiões, desde o catolicismo, passando pela afrodescendente até o islamismo, o prolongamento da vida por meios artificiais é condenado quando todas as possibilidades terapêuticas se esgotaram. No budismo, por exemplo, é aceito que o paciente rejeite alimentos e terapias que não melhorarão seu estado clínico, e mais importante do que prolongar a vida, é vivê-la com qualidade (Chakraborty et al. 2017).

Pacientes gravemente doentes necessitam da intervenção nutricional para não evoluírem para a desnutrição. A doença leva à alteração do metabolismo e consequentemente, em casos crônicos, à caquexia. A utilização da alimentação artificial, por meio de sondas tem por objetivo alcançar a meta energética, uma vez que na maioria das vezes a ingestão via oral está dificultada o que pode gerar desconforto ao paciente. Para os pacientes críticos em estado terminal a priorização dos cuida- 
dos paliativos e a identificação de medidas fúteis devem ser estabelecidas de forma consensual pela equipe multiprofissional, paciente (se capaz), seus familiares ou seu representante legal. Entende-se por medidas fúteis toda intervenção que não atenda ou que seja incoerente com os objetivos propostos no tratamento de um determinado doente. Ao longo de todo o tratamento de um paciente terminal muitas das medidas curativas/restaurativas podem ser entendidas como tratamento fútil como por exemplo: nutrição parenteral ou enteral, administração de drogas vasoativas, terapia renal substitutiva, instituição ou manutenção de ventilação mecânica invasiva e, inclusive, a internação ou permanência do paciente na UTI (Moritz et al. 2008; Fonseca et al. 2012; Mazutti et al. 2016).

Em 1983, o presidente da comissão de estudo sobre problemas éticos em medicina e biomedicina e pesquisa comportamental não encontrou distinção entre nutrição artificial e outros tratamentos de manutenção da vida (President's Commission for the Study of Ethical Problems in Medicine and Biomedical e Behavioral Research 1983). A nutrição artificial é considerada como um tratamento médico e o médico deve tomar decisões de acordo com sua utilidade (Löbbe, 2009). Apesar do impacto positivo da tecnologia, a utilização generalizada deste tratamento no final de vida, estado vegetativo persistente, comprometimento cognitivo grave e demência progressiva avançada cria um dilema ético para alguns que acreditam que a retirada da nutrição artificial é cruel, desumana e leva o paciente à fome (Monturo, 2010).

Pacientes com doenças terminais alcançam um estado de hidratação adequada com muito menos fluido que um paciente hígido necessitaria, e a hidratação exagerada bem como a nutrição artificial podem causar desconforto em pacientes terminais. Eles podem apresentar risco aumentado para bronco-aspiração e pneumonias, diarreia, desconforto gastrointestinal, entre outros. A terapia de hidratação intravenosa em pacientes terminais pode levar a vômitos e à necessidade de sonda nasogástrica de alívio, distúrbios respiratórios com edema pulmonar, edema periférico e aumento da produção urinária, com necessidade de cateterização. Todos esses fatores causam desconforto agudo em pacientes próximos da morte e um estresse emocional nos familiares que acompanham esse processo (Geppert et al. 2010; Maillet et al. 2013). Diante desse cenário, os pacientes e familiares devem decidir entre a retenção ou a retirada do suporte nutricional. Essa decisão é um dilema ético que deve levar em consideração os princípios de autonomia, beneficência, não-maleficência e justiça. Autonomia tem como ideal que a escolha seja feita pelo próprio paciente. A bene- 
ficência refere-se ao alívio do sofrimento do paciente, a não-maleficência prega não prejudicar e a justiça tem por objetivo trazer conforto à vida restante desse paciente (Chernoff 2006; Geppert et al. 2010; Palecek et al. 2010; Snyder et al. 2013).

O direito de um paciente adulto de aceitar ou recusar tratamento médico, incluindo a hidratação e nutrição artificias, está bem estabelecido na legislação e na ética anglo-americanas (Maillet et al. 2002; Ganzini, 2006; Bankhead et al. 2009; Wall, 2011). A doutrina e a prática do consentimento informado são a expressão deste direito à autodeterminação na tomada de decisões em saúde. Legal e eticamente, um adulto munido de informações sobre seu diagnóstico e evolução de sua doença, sem alterações cognitivas, pode optar por manter ou retirar NHA, mesmo que essa escoIha resulte na morte (Jones, 2007; Orrevall, 2015).

Quando se considera um paciente terminal torna-se relevante a discussão sobre as Diretivas Antecipadas de Vontade (DAV). Tais diretivas compõem um documento legal escrito pelo próprio paciente que especifica o tipo de assistência que este deseja receber em situação de terminalidade da vida quando não tiver capacidade para tomar decisões, preservando assim sua autonomia (Bastos e Lerch Lunardi, 2015).

O consentimento livre e esclarecido adequado requer 3 elementos essenciais: (1) que o paciente, familiar ou responsável seja provido de informação médica adequada; (2) que o paciente, familiar ou responsável possua capacidade decisória; e (3) que o paciente ou responsável seja capaz de exercer o voluntariado (a capacidade de tomar uma decisão sem coerção). Informações adequadas incluem, mas não estão limitadas ao diagnóstico, prognóstico, a natureza da intervenção proposta (riscos e benefícios da NHA) e tratamentos alternativos, inclusive a opção de não fazer nada (Appelbaum e Grisso 1988). Caso estes três passos tenham sido cumpridos, podemos dizer que a equipe médica e multiprofissional que assiste o paciente agiu dentro dos princípios bioéticos de beneficência e não maleficência.

Quando se fala em alimentação é impossível dissociá-la da cultura já que o alimento faz parte da formação do complexo processo de formação de identidade cultural de um povo. A comensalidade permeia todas as relações sociais apresentando sempre uma dimensão cultural. Analisando a lógica da comensalidade brasileira, no ato de comer estão implícitas duas situações: 'eu como para viver' e 'eu vivo para comer' (Dias et al. 2015). No primeiro caso está explicito o papel biológico, uma questão de sobrevivência, sendo um fator insubstituível para a manutenção da vida e condi- 
ção sine qua non para todos os seres humanos. Relaciona-se diretamente à vitalidade do indivíduo, à necessidade fisiológica de ingerir nutrientes capazes de manter o corpo em funcionamento, sendo, sob esse aspecto, um comportamento relativo à natureza humana(Lima et al. 2015). Já no segundo caso, o ato de comer e a própria comida refletem os aspectos morais e simbólicos, se relacionando diretamente com a vida social do indivíduo (Maciel, 2005).

Nesse contexto, é importante distinguir o ato de alimentar-se e o de nutrir-se. $\mathrm{O}$ ato de alimentar-se diz respeito à significação no contexto de um universo imaginário e simbólico. $\mathrm{O}$ ato de nutrir-se leva em conta a composição nutricional dos alimentos e seus efeitos fisiológicos no organismo (Silva et al. 2016).

Toda essa contextualização do ato de comer mostra a dificuldade na adoção de restrição da terapia nutricional em pacientes terminais, uma vez que a terapia nutricional raramente cumprirá seu papel na recuperação da saúde desses pacientes. Normalmente, a família ou responsável legal pelo paciente terminal associa os alimentos com saúde e dessa maneira a sua privação significa atentar contra a vida (Bachmann et al. 2001) e não poder ou não conseguir se alimentar significa, em muitos casos, simplesmente a evolução da doença. A idéia da abstinência alimentar desencadeia um sofrimento adicional para todos os envolvidos, uma vez que tem como impacto, além da piora da condição física, conseqüências psicossociais que podem comprometer a qualidade de vida do paciente terminal (Hopkinson et al. 2006; Rhondali et al. 2012). Refletir sobre questões de alimentação relacionadas à finitude sempre provoca polêmicas e opiniões divergentes entre familiares e até mesmo entre profissionais de saúde. A suspensão de NHA encontra resistência da família, pois, apesar de comprovado que o paciente não sente fome nem sede, a família sofre pelo simbólico, por imaginar que seu ente querido morreu de fome e de sede (Pessini, 2004). Esquecem-se que o paciente na realidade está morrendo devido à evolução da sua doença de base, que inclusive provoca anorexia e em alguns casos, a impossibilidade de alimentar-se por meios naturais. Dessa maneira, não só a família como também os próprios profissionais de saúde muitas vezes optam pela manutenção da $\mathrm{NHA}$, apesar de todas as implicações e impactos negativos que isso possa acarretar na qualidade de vida do paciente.

No caso de pacientes terminais, a literatura atual não recomenda o uso de NHA (Swedish Council on Health Technology Assessment in Health Care, 2008; Barrocas et al. 2010; Schwartz et al. 2013, 2014; Druml et al. 2016). A alimentação oral assistida cuidadosa é o método adequado de alimentação, a menos que o estado cognitivo do paciente não permita este tipo de alimentação. O ideal é que o paciente seja 
orientado, enquanto ainda tem poder de decisão, para deixar registrado, através das diretivas antecipadas de vontade, o que deseja que seja feito. Incentiva-se também que os familiares e representantes legais sejam munidos de toda a informação sobre a doença e sua evolução; que estejam cientes de que a doença terminal é que levará o paciente à morte e que a NHA não afetará a progressão da doença; que as decisões sejam tomadas de forma compartilhada entre a equipe de saúde e os familiares ou representante legal do paciente (Schwartz, 2018); e, ainda, que a ausência de NHA não antecipará a morte do paciente. O quadro a seguir apresenta uma compilação dos achados atuais referentes à utilização da NHA em pacientes terminais. Como é possível observar, prevalece a orientação da não utilização desse procedimento.

\begin{tabular}{|c|c|}
\hline Artigo & Conceitos/ Decisões Pertinentes/ Conclusões \\
\hline $\begin{array}{l}\text { Sampson et al. } \\
\text { Enteral tube feeding } \\
\text { for older people } \\
\text { with advanced } \\
\text { dementia. } \\
\text { Cochrane Database } \\
\text { Syst Rev.2009 }\end{array}$ & $\begin{array}{l}\text { Implicações para a Prática: } \\
\text { Apesar do grande número de pacientes que recebem essa } \\
\text { intervenção, há evidências insuficientes. } \\
\text { Não houve evidência de aumento da sobrevida em pacientes que } \\
\text { receberam a alimentação por sonda enteral. Nenhum dos estudos } \\
\text { examinou a QV e não houve evidência de benefício em termos do } \\
\text { estado nutricional ou da prevalência de úlceras por pressão. }\end{array}$ \\
\hline $\begin{array}{l}\text { Barrocas A, et al. } \\
\text { A.S.P.E.N. ethics } \\
\text { position paper. Nutr } \\
\text { Clin Pract. } 2010 .\end{array}$ & $\begin{array}{l}\text { 1- A NHA não fornece nenhum benefício e pode ter riscos } \\
\text { associados em pacientes com demência grave. } \\
2 \text { - Muitos estados nos EUA exigem "evidências claras e } \\
\text { convincentes" para não administrar à NHA em pacientes com } \\
\text { incapacidade decisória sem preferências documentadas de NHA. } \\
3 \text { - Para pacientes sem capacidade de decisão, o profissional de } \\
\text { saúde tem a obrigação ética e legal de fazer referência a uma } \\
\text { Diretivas Antecipadas ou discussão com o tomador de decisão } \\
\text { substituto autorizado, seja ele designado por meio de mecanismos } \\
\text { de uma procuração duradoura para cuidados de saúde, processos } \\
\text { judiciais ou estatutários. } \\
4 \text { - Os tomadores de decisão substitutos (incluindo, mas não se } \\
\text { limitando a, membros da família e/ou outros significativos) devem } \\
\text { receber as mesmas considerações que pacientes individuais com } \\
\text { capacidade de tomar decisões. }\end{array}$ \\
\hline
\end{tabular}




\begin{tabular}{|c|c|}
\hline $\begin{array}{l}\text { Hanson LC. Tube } \\
\text { feeding versus } \\
\text { assisted oral } \\
\text { feeding for persons } \\
\text { with dementia: } \\
\text { using evidence to } \\
\text { support decision- } \\
\text { making. Ann } \\
\text { Longterm Care } \\
2013\end{array}$ & $\begin{array}{l}1 \text { - A literatura apoia a visão de que a alimentação por sonda de } \\
\text { PEG em pacientes com demência não é benéfica em termos de } \\
\text { prevenção da mortalidade ou melhora da qualidade de vida. } \\
\text { 2- A alimentação oral assistida é melhor aceita tanto pelos } \\
\text { pacientes como por suas famílias durante os meses de vida em } \\
\text { declínio dos pacientes. } \\
3 \text { - Após revisar o conjunto de evidências, é aconselhável que } \\
\text { os médicos ofereçam aconselhamento eficaz às famílias de } \\
\text { pessoas com demência avançada, ajudando a apoiar a escolha da } \\
\text { alimentação oral assistida no estágio tardio da doença. }\end{array}$ \\
\hline $\begin{array}{l}\text { tubes in } \\
\text { dementi } \\
\text { statem } \\
\text { Geriatr }\end{array}$ & $\begin{array}{l}1 \text { - Quando surgem dificuldades, os tubos de alimentação não são } \\
\text { recomendados para adultos mais velhos com demência avançada. A } \\
\text { alimentação manual cuidadosa deve ser oferecida porque a alimentação } \\
\text { manual tem se mostrado tão boa quanto a alimentação por sonda para } \\
\text { os resultados de morte, pneumonia por aspiração, estado funcional e } \\
\text { conforto. } \\
\text { Além disso, a alimentação por sonda está associada a agitação, maior } \\
\text { uso de restrições físicas e químicas, uso de cuidados de saúde devido a } \\
\text { complicações relacionadas ao tubo e desenvolvimento de novas úlceras } \\
\text { de pressão. } \\
2 \text { - Os esforços para melhorar a alimentação oral, alterando o ambiente } \\
\text { e criando abordagens centradas no paciente para a alimentação, devem } \\
\text { fazer parte do tratamento usual para idosos com demência avançada. } \\
3 \text { - A alimentação por sonda é uma terapia médica que o tomador de } \\
\text { decisão substituto de um indivíduo pode recusar ou aceitar de acordo } \\
\text { com diretivas antecipadas, desejos previamente declarados ou com o } \\
\text { que se pensa que o indivíduo desejaria. } \\
4 \text { - É responsabilidade de todos os membros da equipe de saúde } \\
\text { que cuidam dos residentes em ambientes de cuidados prolongados } \\
\text { compreender quaisquer desejos anteriormente expressos pelos } \\
\text { indivíduos (através da revisão de diretivas antecipadas e com os } \\
\text { substitutos dos cuidadores) em relação à alimentação por sonda e } \\
\text { incorporar estes desejos. no plano de cuidados. } \\
5 \text { - Instituições como hospitais, casas de repouso e outros ambientes } \\
\text { de cuidados devem promover escolhas, endossar tomadas de decisão } \\
\text { compartilhadas e informadas e honrar as preferências em relação à } \\
\text { alimentação por sonda. Eles não devem impor obrigações ou exercer } \\
\text { pressão sobre indivíduos ou fornecedores para instituir a alimentação } \\
\text { por sonda. }\end{array}$ \\
\hline
\end{tabular}


1 - A decisão de suspender ou interromper a alimentação por sonda na doença terminal é apoiada por evidências científicas atuais

2 - A demência avançada deve ser vista pela equipe de saúde como uma doença terminal, e os membros da equipe de saúde devem comunicar claramente essa perspectiva à família do paciente, a outros significativos, aos cuidadores e / ou aos tomadores de decisão substitutos.

3 - Uma discussão completa deve ocorrer com o paciente, a família, os outros significativos, cuidadores e / ou tomadores de decisão substitutos. A conversa deve cobrir as descobertas baseadas em evidências mais atualizadas em relação a riscos, encargos e benefícios de curto e longo prazo.

Schwartz DB, et al.

4 - Alternativas como a alimentação oral assistida e outras A.S.P.E.N. special intervenções orais inovadoras devem ser amplamente exploradas report: gastrostomy tube placement in patients with advanced dementia or near end of life.

Nutr Clinc Prac.

2014 e discutidas com o paciente, a família, os outros significativos, os cuidadores e / ou os tomadores de decisão substitutos.

5. A autonomia do paciente ou tomador de decisão substituto deve ser respeitada. A ênfase deve ser colocada no status funcional e na qualidade de vida. Um aspecto essencial do processo envolve sensibilidade cultural, religiosa, social e emocional ao sistema de valores do paciente. Um estudo de tempo limitado de alimentação nasogástrica pode ser considerado se for tomada uma decisão de prosseguir no futuro com um tubo-G.

6 - A decisão informada final deve ser alcançada por meio de uma abordagem, incluindo familiares, significativos, cuidadores e / ou tomadores de decisão substitutos.

7 - Os médicos das instituições de saúde, tanto hospitais como instituições de longa permanência, devem desenvolver um processo que seja interdisciplinar, colaborativo, proativo, integrado e sistemático, a fim de facilitar a tomada de decisões que envolvam o paciente, a família, os outros, cuidadores, e/ou tomadores de decisão substitutos. O processo deve promover a DA que ofereça cuidados de saúde com base nos desejos e interesse do paciente 
Druml C, et al. ESPEN guideline on ethical aspects of arti cial nutrition and hydration. Clin Nutr. 2016
1 - Se os riscos/encargos de uma terapia superam os potenciais benefícios, o médico tem a obrigação de não fornecer (reter) a terapia.

2 - A terapia nutricional para pacientes idosos é frequentemente destinada a garantir um suprimento permanente de nutrição $e$ hidratação até fim de vida. A justificativa para tal tratamento deve ser revisada criticamente em intervalos regulares.

3 - Para pacientes com demência avançada, a prioridade deve ser sempre dada à assistência alimentar cuidadosa/ alimentação manual.

4 - Mesmo que o paciente não seja legalmente competente de acordo com a lei civil, ele ainda pode ser capaz de expressar seus desejos e participar do processo de tomada de decisão.

5 - No caso de um paciente ser incapaz de dar consentimento e fazer julgamentos, o seu representante legal (dependendo da lei e prática do país) toma a decisão. Se a decisão dos representantes demorar muito, o médico deve iniciar a nutrição artificial de acordo com a indicação médica baseada em evidências.

6 - Na ausência de uma declaração efetiva da vontade do paciente em uma situação específica, deve-se proceder de acordo com a vontade presumida do paciente. $O$ representante legal do paciente é obrigado a determinar a vontade presumida do paciente.

7 - A Qualidade de vida deve ser sempre considerada em qualquer tipo de tratamento médico, incluindo nutrição artificial.

8 - Um tratamento médico, que não oferece nenhum benefício ou se tornou desproporcional pode ser retirado ou retido.

9 - Fornecer nutrição contra a vontade do paciente que é capaz de dar o seu consentimento ou fazer julgamentos (alimentação forçada) é geralmente proibida.

Fonte: Traduzido de Schwartz DB, 2018.

Considerando as informações contidas em diversos artigos em especial os relacionados com as recomendações de órgãos oficiais, foi possível observar que estes não indicam a utilização da NHA em pacientes terminais, sugerem o uso de alimentação manual assistida como alternativa, e reiteram a importância de respeito a autonomia do paciente, através das diretivas antecipadas de vontade, e quando este 
instrumento não estiver disponível deve-se proceder de acordo com a vontade presumida do paciente, relatada pelos familiares ou representante legal deste. Ressaltam também a importância da decisão compartilhada entre equipe de saúde e familiares ou representante legal.

Pacientes com doenças terminais apresentam um menor apetite e dependendo da condição subjacente, podem não conseguir comer e como consequência, evoluem para a desidratação e subnutrição caso a intervenção não aconteça. Faz parte do senso comum que a morte com desidratação e desnutrição leve ao sofrimento desnecessário do paciente. Porém, de acordo com a experiência de profissionais da saúde que acompanharam pacientes terminais, aqueles que se encontravam desidratados experimentam melhorias nos sintomas, como alívio das sensações de engasgamento e afogamento, com menor tosse e congestão à medida que ocorre a diminuição das secreções pulmonares; diminuição da produção de urina com menor necessidade de cateterismo e menos episódios de enurese; diminuição do líquido gastrointestinal com menor número de vômitos, menor inchaço e diarreia; diminuição do edema periférico e consequentemente uma morte mais confortável. A maioria desses pacientes não tem fome ou sede (Andrews et al. 1993; Fine, 2006).

Os médicos e outros profissionais envolvidos com os cuidados de pacientes em estado terminal devem estar preparados para a discussão sobre continuar ou retirar certas modalidades terapêuticas que aumentam o processo de morte. O papel do médico nos cuidados com o final da vida é bastante complexo. Muitos presumem que o alvo primário é preservar ou restaurar a vida, porém o médico deve endereçar sua atenção ao conforto do paciente, incluindo analgesia, alívio de outros sintomas como dispnéia, náuseas, fadiga e depressão (Marco e Schears 2006; Hales et al. 2010; Cook et al. 2015). Assim, torna-se fundamental o cultivo de três elementos no cuidado: a compaixão, a humildade e a honestidade, em contexto e visão multidisciplinares. O trabalho com o paciente terminal exige maturidade e conhecimento sobre a posição adotada diante da morte e do morrer.

Os conceitos de eutanásia, distanásia e ortotanásia embora sejam aparentemente semelhantes, expressam significados distintos. O termo eutanásia deriva de uma palavra composta de origem grega, sendo eu (bem) e thanatos (morte), significando boa morte, ou morte tranquila, doce e sem sofrimentos. O conceito prevalente de eutanásia é a antecipação da morte de paciente incurável, geralmente terminal e em grande sofrimento físico ou psíquico, movido por compaixão. Ela pode ser classifica- 
da, segundo o modo de atuação do agente, em eutanásia ativa e eutanásia passiva; em relação à intenção do profissional, em direta e indireta ou duplo-efeito; e segundo a vontade do paciente, em voluntária e involuntária (Siqueira-Batista e Schramm 2004).

Segundo a definição do dicionário Houaiss, distanásia significa morte lenta, com grande sofrimento (2001). Trata-se, assim, de um neologismo, uma palavra nova, de origem grega. O prefixo grego dis (afastamento), portanto a distanásia significa prolongamento exagerado da morte de um paciente. A distanásia é conceituada como agonia prolongada, morte com sofrimento físico ou psicológico do indivíduo lúcido e utilização de procedimentos fúteis que levam ao prolongamento de vida. A futilidade terapêutica pode ser definida como o conjunto de procedimentos diagnósticos ou terapêuticos inadequados e inúteis diante da situação irreversível da doença que pode causar sofrimento ao doente e a seus familiares ou terapia que não é capaz de atingir seus objetivos e não aumente a sobrevida e melhore a qualidade de vida (Ardagh, 2000).

A ortotanásia tem seu nome proveniente dos radicais gregos: orthos (reto, correto) e thanatos (morte), indica assim, a morte a seu tempo, ou a morte no tempo correto, nem antes nem depois da hora. A ortotanásia se apresenta como uma nova modalidade de assistência para "uma boa morte", traduzindo a morte como um processo humanizado e oferecendo conforto sem o prolongamento abusivo da vida por meios artificiais ainda é objeto de muita discussão e nem sempre o médico tem seu conceito claro. De acordo com Vasconcelos, Imamura e Villar, foi observado que dentre os conceitos de ortotanásia, distanásia e eutanásia, o de ortotanásia gerou maior confusão conceitual para os profissionais da saúde (Vasconcelos et al. 2011). Muitas vezes, para os profissionais de saúde, a definição de ortotanásia se confunde com a de eutanásia, ou seja, é entendida uma morte piedosa, evidenciando a necessidade de se difundir tais conceitos para aqueles que lidam com a morte no seu dia-a-dia (Batista e Seidl 2011). Do ponto de vista jurídico, trata-se da diferença entre matar e deixar morrer. Do ponto de vista médico, trata-se da diferença entre esforço terapêutico e cuidados paliativos.

De acordo com alguns autores, a ortotanásia se encontra entre a eutanásia e a distanásia, uma vez que auxilia o paciente a entender a sua finitude, alivia o sofrimento físico, espiritual e emocional e prepara o indivíduo para o processo de morrer. Com a humanização da morte, o paciente terminal tem a chance de se aproximar dos entes queridos proporcionando uma despedida sem culpas e dúvidas (Siqueira et al. 2013). 
Com a finalidade de auxiliar e suportar os médicos, o Conselho Federal de Medicina (CFM) editou a Resolução 1.805/06 (Conselho Federal de Medicina, 2006) que autoriza esses profissionais a limitarem ou suspenderem procedimentos e tratamentos que prolonguem a vida do doente em fase terminal, de enfermidade grave $e$ incurável, respeitada a vontade da pessoa ou de seu representante legal (Sanchez Y Sanches KM e Seidl, 2013). Segundo o Código de Ética Médica (CEM) a eutanásia não deve ser praticada e a distanásia é desencorajada.

O médico, ao praticar a ortotanásia, não estaria agindo com negligência, imperícia ou imprudência. Isso porque, para que a ortotanásia se configure, é preciso que o médico avalie o prognóstico do paciente, sempre levando em consideração a qualidade da sua morte e autonomia. Além disso, o médico deve buscar conversar com o paciente sobre o fim da vida, os possíveis tratamentos e o encaminhe para o cuidado paliativo (Cruz e Oliveira 2013). Nesse contexto não se pode atribuir a morte do doente terminal à conduta do médico que pratica a ortotanásia, especialmente porque além de não privá-lo dos cuidados essenciais, evitou procedimentos fúteis.

\section{Discussão}

No cenário da terminalidade, é imprescindível a reflexão sobre o processo de cuidar. A sua humanização toma o lugar de uma assistência técnica, permitindo a compreensão das situações do cotidiano assistencial no cuidar no processo de morrer. Nas instituições hospitalares o processo de humanização é imprescindível e primeiramente pressupõe o entendimento do significado da vida do ser humano, sendo parte do cuidar humanizado o seu acolhimento físico, psicológico e espiritual, encerrando o homem em toda a sua complexidade (Marengo et al. 2009).

As questões éticas que cercam o alimento e o ato de alimentar são complexas, uma vez que a comida e a bebida têm funções psicológicas e fisiológicas que geralmente desempenham um papel essencial no cuidado do indivíduo. $O$ alimento tem fortes implicações emocionais e simbólicas que incluem nutrição materna e valores religiosos, culturais e sociais. Para que se alcance uma visão humanizada do paciente terminal, é necessário reconhecer que cada pessoa aborda o fim da vida com diferentes atitudes e valores culturais, religiosos e pessoais. Para algumas pessoas, todo momento de vida, não importa quão doloroso e limitado, é de valor inestimável. Outras pessoas podem tentar renunciar a várias terapias médicas que podem incluir apoio nutricional. No entanto, outros podem recusar procedimentos médicos, como a 
ventilação mecânica, mas desejam continuar a nutrição ou o suporte de hidratação. As tradições religiosas e culturais podem afirmar que sustentar a vida é uma obrigação moral, com o contra-argumento de que, se não houver benefício, o procedimento não pode ser obrigatório (Geppert et al. 2010).

Quando há discordância sobre a futilidade dos tratamentos, surge a questão de quando a nutrição e a hidratação é moralmente obrigatória ou moralmente opcional. Um método comum de distinguir entre o que é obrigatório e o que é opcional é considerar as consequências. Nutrição e hidratação podem ser eficazes na medida em que mantêm a vida, mas por si mesmas não podem restaurar a consciência, reverter as comorbidades relacionadas a doença ou impedir a morte. $O$ respeito pela autonomia significa permitir que o paciente tome suas próprias decisões. A competência do paciente para tomar decisões é relevante aqui, e se isso for prejudicado, as declarações anteriores feitas quando competentes (como testamento vital ou diretiva antecipada) podem ser levadas em consideração. A concepção moderna da atenção à saúde centrada no paciente significa que a autonomia é agora considerada o princípio mais importante da ética médica. Isso tem sido criticado como dogmatismo ocidental, uma vez que outras culturas podem não valorizar a autonomia na mesma proporção (Ferrie, 2006). No cenário da terminalidade, é imprescindível a reflexão sobre o processo de cuidar. A sua humanização toma o lugar de uma assistência técnica, permitindo a compreensão das situações do cotidiano assistencial no cuidar no processo de morrer.

A questão da justiça e da alimentação individual pode se intensificar, especialmente para os permanentemente inconscientes. Essa condição de vulnerabilidade do paciente transfere a responsabilidade da tomada de decisão em alimentar ou não, para o responsável legal ou familiares. Um dos primeiros sinais de agravamento da doença é a perda do apetite, o que causa muita angústia aos familiares e nesse contexto, os profissionais envolvidos no tratamento precisam estar aptos a identificar os benefícios da alimentação por via oral, enteral e/ou parenteral, no que tange a qualidade de vida do paciente. No caso do paciente que está impossibilitado de se comunicar por coma, rebaixamento do nível de consciência, confusão mental ou demência, os profissionais responsáveis devem considerar a opinião da família e definir com ela qual a melhor conduta a ser adotada. É da responsabilidade do clínico solicitar e compreender as metas e valores previamente definidos pelo paciente e, em seguida, orientar e facilitar as decisões médicas para que os tratamentos fornecidos sejam consistentes com esses objetivos e valores. Também é importante reconhecer 
que as decisões de manter ou suspender a terapia para prolongamento da vida podem ser particularmente estressantes para os membros da família. Para minimizar o ônus da tomada de decisão dos familiares ou responsável legal, o médico deve estar preparado para promover evidências compassivas, aconselhar e buscar chegar a um consenso para que a decisão seja compartilhada ou acordada. Pacientes, familiares e médicos muitas vezes lutam com a questão de saber se a suspensão ou a retirada da NHA é equivalente a "matar" pacientes. O objetivo de manter ou suspender a NHA não é acabar com a vida do paciente e sim "deixa-lo ir". É importante tranquilizar os membros da família de que interromper a NHA não significa abandonar o paciente e sim que o foco agora será no gerenciamento de sintomas "ativos". As famílias podem se sentir menos culpadas se lhes for dito que cuidados de baixa tecnologia e alto contato serão fornecidos (Arcand, 2015). A administração de fluidos e energia nem sempre é necessária em todos os momentos nesta fase da vida. Os pacientes frequentemente experimentam secura da boca, sensação precoce de saturação, náusea e o paladar alterado, mas raramente fome e sede. A sede geralmente resulta do ressecamento da cavidade oral e da formação de crostas e pode ser aliviada por cuidados orais e pequenas quantidades de líquidos, menos do que o necessário para aliviar a desidratação. A administração parenteral de fluido não necessariamente alivia a sede do indivíduo (Slomka, 2003). Além disso, o ressecamento da boca pode ser um efeito colateral da medicação, oxigenoterapia, respiração pela boca ou ansiedade e depressão. No caso raro de um paciente estar com sede apesar dos cuidados ideais ou quando a desidratação está associada ao delirium, a eficácia da hidratação artificial pode ser revista, mas é duvidosa na fase de morrer (Bruera et al. 2013; Good et al. 2014).

\section{Conclusão}

Fatores religiosos, culturais e a aceitação da finitude são muito importantes e influenciam também a tomada de decisão e o modo de agir do médico e dos familiares do paciente ou seu representante legal. Além desses fatores, o médico deve ter consciência de que a prescrição da NHA é considerada ato médico e deve ter conhecimento de toda a literatura científica a este respeito, e deve guiar-se por eles. Caso os riscos/encargos de uma terapia superem os potenciais benefícios, o médico tem a obrigação de não fornecer esta terapia. $\mathrm{E}$, ainda, todas as suas ações devam ser pautadas pelos princípios bioéticos da autonomia do paciente, beneficência e 
não maleficência, utilizados na definição junto ao paciente, seus familiares ou representante legal das terapias a serem instituídas terminalidade de vida, o princípio da justiça tem lugar especial neste momento pois o paciente necessitará de todo o apoio do sistema de saúde no tratamento de sua doença.

Nutrir o doente terminal tem mais um significado simbólico do que fisiológico e na maioria das vezes essa prática causa desconforto e piora clínica. Cuidar, controlar os sintomas buscando a melhor qualidade de vida possível nesta fase deve ser a meta da equipe médica e multiprofissional. A ortotanásia é uma prática que vai ao encontro do que se preconiza como uma morte digna: permitir que o paciente morra da doença sem dor ou desconforto e respeitando o tempo de evolução do seu quadro clínico. Esse trabalho propôs uma reflexão à luz da bioética, sobre a importância do cuidar quando a cura é impossível e reconhecer a terminalidade como processo humano, natural e necessário.

\section{Referências}

1. Alsolamy S. Islamic views on artificial nutrition and hydration in terminally III patients. Bioethics. 2014;28(2):96-9.

2. Andrews M, Bell E, Smith S, Tischler J, Veglia J. Dehydration in terminally ill patients. Is it appropriate palliative care? Postgrad Med. 1993;93(1):201-3, 206-8.

3. Appelbaum PS, Grisso T. Assessing Patients' Capacities to Consent to Treatment. N Engl J Med [Internet]. 1988;319(25):1635-8. Available from: http://www. nejm.org/doi/abs/10.1056/NEJM198812223192504

4. Arcand M. End-of-life issues in advanced dementia: Part 1: goals of care, decision-making process, and family education. Can Fam Physician. 2015;61(4):3304.

5. Ardagh M. Futility has no utility in resuscitation medicine. J Med Ethics. 2000;26:396-9.

6. Bachmann P, Marti-Massoud C, Blanc-Vincent MP, Desport JC, Colomb V, Dieu L, et al. Standards, options et recommandations: Nutrition en situation palliative ou terminale de l'adulte porteur de cancer ??volutif. Bull Cancer. 2001;88(10):9851006.

7. Bankhead R, Boullata J, Brantley S, Corkins M, Guenter P, Krenitsky J, et al. A.S.P.E.N. Enteral Nutrition Practice Recommendations. J Parenter Enter Nutr [Internet]. 2009;33(2):122-67. Available from: http://journals.sagepub.com/ doi/10.1177/0148607108330314

8. Barrocas A, Geppert C, Durfee SM, Sullivan JO, Rd M, Monturo C, et al. A.S.P.E.N. Ethics Position Paper. Nutr Clin Pr. 2010;25(6):672-9. 
9. Bastos S, Lerch Lunardi V. Diretivas antecipadas de vontade aos doentes terminais: revisão integrativa. Rev Bras Enferm. 2015;68(3):524-58.

10. Batista K, Seidl E. Estudo acerca de decisões éticas na terminalidade da vida em unidade de terapia intensiva. Com Ciências Saúde. 2011;22(1):51-60.

11. Bitencourt AGV, Neves FBCS, Durães L, Nascimento DT, Neves NMBC, Torreão LDA, et al. Avaliação do conhecimento de estudantes de medicina sobre morte encefálica. Rev Bras Ter Intensiva. 2007;19(2):144-50.

12. Bruera E, Hui D, Dalal S, Torres-Vigil I, Trumble J, Roosth J, et al. Parenteral hydration in patients with advanced cancer: a multicenter, double-blind, placebo-controlled randomized trial. J Clin Oncol. 2013;31(1):111-8.

13. Chakraborty R, El-Jawahri AR, Litzow MR, Syrjala KL, Parnes AD, Hashmi SK. A systematic review of religious beliefs about major end-of-life alliat Support Care issues in the five major world religions. Palliat Support care. 2017;25(4):368-79.

14. Chernoff R. Tube feeding patients with dementia. Nutr Clin Pract. 2006;21(2):142-6.

15. Cook D, Swinton M, Toledo F, Clarke F, Rose T, Hand-Breckenridge T, et al. Personalizing death in the intensive care unit: The 3 wishes project a mixed-methods study. Ann Intern Med. 2015;163(4):271-9.

16. Cruz MLM da, Oliveira RA de. A licitude civil da prática da ortotanásia por médico em respeito à vontade livre do paciente. Rev Bioética. 2013;21(3):405-11.

17. Dias A, Matos R, Braga D, Magossi A, Diniz A, LS A. Riscos Ocupacionais em Atividade de Coleta de Resíduos Sólidos Occupatio. 2015;1:3-17.

18. Druml C, Ballmer PE, Druml W, Oehmichen F, Shenkin A, Singer P, et al. ESPEN guideline on ethical aspects of artificial nutrition and hydration. Clin Nutr. 2016;35(3):545-56.

19. Ferrie S. A quick guide to ethical theory in healthcare: solving ethical dilemmas in nutrition support situations. Nutr Clin Pract. 2006;21(2):113-7.

20. Fine RL. Ethical issues in artificial nutrition and hydration. Nutr Clin Pr [Internet]. 2006;21(2):118-25. Available from: http://www.ncbi.nlm.nih.gov/pub$\mathrm{med} / 16556921$

21. Firth S. End-of-life: A Hindu view. Vol. 366, Lancet. 2005. p. 682-6.

22. Fonseca AC, Mendes Junior W V., Fonseca MJM. Cuidados paliativos para idosos na unidade de terapia intensiva: revisão sistemática. Rev bras ter intensiva. 2012;24(2):197-206.

23. Ganzini L. Artificial nutrition and hydration at the end of life: ethics and evidence. Palliat Support Care [Internet]. 2006;4(2):135-43. Available from: http://www.ncbi. nlm.nih.gov/pubmed/16903584

24. Geppert CMA, Andrews MR, Druyan ME. Ethical Issues in Artificial Nutrition and Hydration: A Review. J Parenter Enter Nutr. 2010;34(1):79-88. 
25. Giacomin KC, Dos Santos WJ, Firmo JO. O luto antecipado diante da consciência da finitude: a vida entre os medos de não dar conta, de dar trabalho e de morrer. Ciência e Saúde Coletiva. 2013;18(9):2487-96.

26. Good P, Richard R, Syrmis W, Jenkins-Marsh S, Stephens J. Medically assisted hydration for adult palliative care patients. Cochrane Database Syst Rev. 2014;(4):1-19.

27. Hales S, Zimmermann C, Rodin G. Review: The quality of dying and death: a systematic review of measures. Palliat Med. 2010;24(2):127-44.

28. Hanson LC. Tube feeding versus assisted oral feeding for persons with dementia: Using evidence to support decision-making. Ann Long-Term Care. 2013;21:36-9.

29. Hopkinson J, Wright $D$, Corner J. Exploring the experience of weight loss in people with advanced cancer. J Adv Nurs. 2006;54(3):304-12.

30. Hui D, Nooruddin Z, Didwaniya N, Dev R, De La Cruz M, Kim SH, et al. Concepts and Definitions for "Actively Dying," "End of Life," "Terminally III," "Terminal Care," and "Transition of Care": A Systematic Review. J Pain Symptom Manage. 2014;47(1):77-89.

31. Jones BJM. Nutritional support at the end of life: the relevant ethical issues. Eur J Gastroenterol Hepatol. 2007;19(5):383-8.

32. Knobel M, Silva ALM. O paciente terminal: vale a pena insistir no tratamento? Einsten. 2004;2(2):133.

33. Kovács MJ. Educação para a Morte. Psicol Ciência e Profissão. 2005;25(3):484-97.

34. Lago PM, Devictor D, Piva JP, Bergounioux J. Cuidados de final de vida em crianças: perspectivas no Brasil e no mundo. J Pediatr (Rio J). 2007;83(2):109-S116.

35. Lima R, Neto J, Farias R. Alimentação, Comida E Cultura: O Exercício Da Comensalidade. Aliment Nutr Saúde. 2015;10(3):507-22.

36. Löbbe V. Nutrition in the last days of life. Curr Opin Support Palliat Care. 2009;3(3):195-202.

37. Maciel M. Olhares antropológicos sobre a alimentação. Canesqui A, Garcia R, editors. Antropol e Nutr um diálogo possível. 2005;

38. Maillet JO, Potter RL, Heller L. Position of the American Dietetic Association: ethical and legal issues in nutrition, hydration, and feeding. J Am Diet Assoc. 2002;102:716-26.

39. Maillet JO, Schwartz DB, Posthauer ME. Position of the academy of nutrition and dietetics: Ethical and legal issues in feeding and hydration. $J$ Acad Nutr Diet. 2013;113(6):828-33.

40. Marco CA, Schears RM. Death, Dying, and Last Wishes. Vol. 24, Emergency Medicine Clinics of North America. 2006. p. 969-87.

41. Marengo M, Flávio $D$, Silva R. Terminalidade de vida : bioética terminalidade e humanização em saúde. Med (Ribeirão Preto). 2009;42(3):350-7. 
42. Mazutti SRG, Nascimento A de F, Fumis RRL. Limitação de Suporte Avançado de Vida em pacientes admitidos em unidade de terapia intensiva com cuidados paliativos integrados. Rev Bras Ter Intensiva. 2016;28(3):294-300.

43. Mitchell BL, Mitchell LC. Review of the literature on cultural competence and end-of-life treatment decisions: the role of the hospitalist. J Natl Med Assoc. 2009;101(9):920-6.

44. Monturo $C$. The artificial nutrition debate: still an issue... after all these years. Nutr Clin Pract. 2010;24(2):206-13.

45. Moritz R. Os profissionais de saúde diante da morte e do morrer. Rev Bioética. 2005;13(2):51-63.

46. Moritz RD, Lago PM Do, Souza RP De, Silva NB Da, Meneses FA De, Othero JCB, et al. Terminalidade e cuidados paliativos na unidade de terapia intensiva. Rev Bras Ter Intensiva. 2008;20(4):422-8.

47. Nasser E. Nietzsche e a morte. Cad Filos Alemã Crítica e Mod. 2008 Jun 14;0(11):99.

48. Neto YC. Morte encefálica : cinquenta anos além do coma profundo. RevBras. saúde Matern Matern. 2010;10(2):355-61.

49. Orrevall Y. Nutritional support at the end of life. Nutrition [Internet]. 2015;31(4):6156. Available from: http://dx.doi.org/10.1016/j.nut.2014.12.004

50. Palecek EJ, Teno JM, Casarett DJ, Hanson LC, Rhodes RL, Mitchell SL. Comfort feeding only: A proposal to bring clarity to decision-making regarding difficulty with eating for persons with advanced dementia. Vol. 58, Journal of the American Geriatrics Society. 2010. p. 580-4.

51. Pessini L. A filosofia dos cuidados paliativos: uma resposta diante da obstinação terapêutica. In: BERTACHINI, L.; PESSINI L, editor. Humanização e cuidados paliativos. 3th ed. São Paulo: Loyola; 2004. p. 181-208.

52. President's Commission for the Study of Ethical Problems in Medicine and Biomedical and Behavioral Research. Deciding to forego life-sustaining treatment: A report on the ethical, medical, and legal issues in treatment decisions. Pres Comm Study Ethical Probl Med Biomed Behav Res. 1983;217-23.

53. Rhondali W, Filbet M, Chisholm GB, Daneshmand MD, Hui D, Fingeret MC. An examination of weight loss and body image concerns for advanced cancer patients and their caregivers. Vol. 26, Palliative Medicine. 2012. p. 433.

54. Ricoeur P. Ética y moral. In: Doce textos fundamentales de la ética del Siglo XX. 2007. p. 241-64.

55. Salviano J. A metafísica da morte de Schopenhauer. ethic@ - An Int J Moral Philos [Internet]. 2012 Sep 29 [cited 2019 Feb 28];11(2):187-97. Available from: http://www.periodicos.ufsc.br/index.php/ethic/article/view/26365 
56. Sampson EL, Candy B, Jones L. Enteral tube feeding for older people with advanced dementia. Cochrane Database Syst Rev [Internet]. 2009 Apr 15 [cited 2019 Aug 13];(2). Available from: http://doi.wiley.com/10.1002/14651858.CD007209.pub2

57. Sanchez Y Sanches KM; Seidl EMF. Ortotanásia: Uma decisão frente à terminalidade. Interface Commun Heal Educ. 2013;17(44):23-34.

58. Schwartz DB. Enteral Nutrition and Dementia Integrating Ethics. Nutr Clin Pract. 2018;33(3):377-87.

59. Schwartz DB, Barrocas A, Wesley JR, Kliger G, Pontes-Arruda A, Márquez HA, et al. Gastrostomy Tube Placement in Patients With Advanced Dementia or Near End of Life. Nutr Clin Pract. 2014;29(6):829-40.

60. Schwartz DB, Posthauer ME, O'Sullivan Maillet J. Practice Paper of the Academy of Nutrition and Dietetics Abstract: Ethical and Legal Issues of Feeding and $\mathrm{Hy}$ dration. J Acad Nutr Diet. 2013;113(7):981.

61. Silva L, Silva M, Sena-Evangelist K, Silva F, Silva B. A boa morte : o holos do " comer" no fim da vida. RAZÓN Y PALABRA. 2016;3(94):695-708.

62. Siqueira-Batista R, Schramm FR. Eutanásia: pelas veredas da morte e da autonomia. Cien Saude Colet. 2004;9(1):31-41.

63. Siqueira JE, Pessini L, Motta De Siqueira CE. Conflitos morais sobre a terminalidade da vida: aspectos médicos, filosóficos e jurídicos. Rev Colomb Bioética. 2013;8(2):104-15.

64. Slomka J. Withholding nutrition at the end of life: Clinical and ethical issues. Cleve Clin J Med. 2003;70(6):548-52.

65. Smith AK, McCarthy EP, Paulk E, Balboni TA, Maciejewski PK, Block SD, et al. Racial and ethnic differences in advance care planning among patients with cancer: impact of terminal illness acknowledgment, religiousness, and treatment preferences. J Clin Oncol. 2008;26(25):4131-7.

66. Snyder EA, Caprio AJ, Wessell K, Lin FC, Hanson LC. Impact of a decision aid on surrogate decision-makers' perceptions of feeding options for patients with dementia. J Am Med Dir Assoc. 2013;14(2):114-8.

67. Souza MT de, Silva MD da, Carvalho R de. Integrative review: what is it? How to do it? Einstein (São Paulo, Brazil). 2010;8(1):102-6.

68. Srinonprasert V, Kajornkijaroen A, Bangchang PN, Wangtrakuldee G, Wongboon$\sin \mathrm{J}$, Kuptniratsaikul V, et al. A survey of opinions regarding wishes toward the end-of-life among Thai elderly. J Med Assoc Thai. 2014;97(3):216-22.

69. Statens beredning för medicinsk utvärdering., Elanders Infologistics Väst). Dementia - etiology and epidemiology: a systematic review : June 2008. Vol. 1. Statens beredning för medicinsk utvärdering (SBU); 2008.

70. Steinhauser KE, Christakis NA, Clipp EC, McNeilly M, Mclntyre L, Tulsky JA. Factors considered important at the end of life by patients, family, physicians, and other care providers. JAMA. 2000;284(19):2476-82. 
71. Torres WDC. A Bioética e a Psicologia da Saúde : Reflexões sobre Questões de Vida e Morte. Medicine (Baltimore). 2003;16(3):475-82.

72. Vasconcelos T, Imamura N, Villar H. Impacto da Resolução CFM 1.805/06 sobre os médicos que lidam com a morte. Rev bioét. 2011;19(2):501-21.

73. Wall A. Code of Medical Ethics of the American Medical Association: Current Opinions With Annotations 2010-2011. Med Sci Sport Exerc. 2011;43(6):1133.

74. van Wijmen MPS, Pasman HRW, Widdershoven GAM, Onwuteaka-Philipsen BD. Continuing or forgoing treatment at the end of life? Preferences of the general public and people with an advance directive. J Med Ethics. 2015;41(8):599-606.

75. Zoboli ELCP, Schveitzer MC. Valores de la enfermería como práctica social : una metasíntesis cualitativa. Rev Latino-Am Enferm. 2013;21(3):2-9.

76. American Geriatrics Society Feeding Tubes in Advanced Dementia Position Statement. J Am Geriatr Soc. 2014;62(8):1590-3.

Recebido: 26/06/2019. Aprovado: 18/08/2019. 